\title{
Effect on Spiral Bevel Gear Tooth Profile
}

\author{
Hongfang Liu \\ College of Mechanical Engineering, Hubei Polytechnic Institute, Xiaogan Hubei, 432000, China
}

Keywords: Machine tool error, Spiral bevel gear, Gear shapes

\begin{abstract}
Machine spiral bevel gear tooth surface will be associated with the machine error in the event of a failure, the mathematical model with the use of the new method of calculation error based on better understanding of spiral bevel gears, the error theory and the law of error flank tooth surfaces calculated. And the Machine Tool and Tool error parameter adjustment errors can be introduced into the study of the influence of different error understand the correlation between the sizes of the error value to provide guidance and basis for the production and processing.
\end{abstract}

\section{Introduction}

As a key part, spiral bevel gear provides support for the rotation of machines, but given the gnashing of teeth, when the theoretical design more complex, cumbersome, in the machining of spiral bevel gears need to consider various factors. LTCA loaded tooth contact analysis technology began to be applied, but LCA, LTCA technology is based on theoretical analysis, there is often a difference between theory and actual tooth surface, whereby the spiral bevel gear error is mainly produced in the machine error, there is a correlation between the two, error magnitude can also be reflected machining accuracy. When the calculation of the profile error, can be combined with theoretical tooth surface grid point vector to form a projection profile error, there is a difference in the CMM profile error detection, and then generate inevitable errors. Accordingly, the latest calculation methods herein profile error based application development profile error analysis software, will introduce errors into the machine, on the influence of errors were analyzed and the relative error of the tooth surface error values deviate from the theoretical tooth surface.

\section{Establish mathematic model of tooth surface}

Gear pair contacts with a conjugate point of spatial pattern, and gear blanks and tool are core components of spatial location and complex relative movement, through this motion determines the geometry of the spiral bevel gear surfaces. This paper built on the basis of vector calculation method will be conical cutter cutting face up, set up at any point in the cut surface $\mathrm{M}$, whose normal vector is $\mathrm{n}$, the unit vector $\mathrm{t}, \mathrm{M}$ running along the generatrix direction, $\mathrm{O}$ come to the origin of the vector equation $r \mathrm{M}$ point, while $\mathrm{O}^{\prime}$ origin vector equation was $r_{c}$. Eventually, $r$ will determine whether the formation of a conical cutting surface angle $\mathrm{q}$ remote station parameters sport surfaces, if the formation of a movement, said conical cutting face surface and a conjugated surface contact can engage the equation, by equation tooth surface origin cradle angle and $\mathrm{M}$ point conjugate contact angle cutter, work corner, respectively, with $\mathrm{q}, \theta, \varphi$ said that the position of the workpiece at the time of the original tooth surface diameter vector was $r_{w}$, France vector $n_{w}$, its formula is expressed as:

$$
\begin{aligned}
& \mathrm{Rw}=(\mathrm{p} \cdot \mathrm{rc}) \mathrm{p}+\cos \varphi(\mathrm{p} \times \mathrm{rc}) \times \mathrm{p}+\sin \varphi(\mathrm{p} \times \mathrm{rc}) \\
& \mathrm{Nw}=(\mathrm{p} \cdot \mathrm{n}) \mathrm{p}+\cos \varphi(\mathrm{p} \times \mathrm{n}) \times \mathrm{p}+\sin \varphi_{(\mathrm{p} \times \mathrm{n})}
\end{aligned}
$$

In the formula, $\mathrm{P}$ represents the axis of the workpiece. 


\section{Discretization of tooth surface}

Space spiral bevel gear tooth surface is more complex surfaces, in order to make theoretical tooth surface and tooth surface error calculation is more reasonable, clear discrete processing will be carried out. At a fixed point flank plane image corresponding to a grid plan, a relatively simple topology map is to do in the tooth surface rotary shaft section projection. In addition, in order to make the analysis results closer gear measuring center or CMM measurements may be correspondence between the established, when meshing on the projection, the need to maintain the grid, middle and lower, left and right of the uniform, and to control the contraction within reasonable limits, you can refer to shrink the size of the gear measuring center distance, can also be laid in accordance with CMM parameters, AGMA standard reference ${ }^{[1]}$. To more realistic tooth surface morphology reflected on the tooth surface can be arranged discrete density, divided into 50 discrete points, combined with a high standard take four rows of teeth. According to the tooth surface and shrink the size of the blank parameter range of different discrete points is calculated, then $O$ 'as the origin of the coordinate center, set (x (i,j), y (i,j), which can be $0-8$ i represents a value from 1 to 6 can represent the value of $\mathrm{j}$. from the gear axis $\mathrm{M} p$ will point to the tooth surface to $\mathrm{y}$, then along the axis of the gear is designed for cross-point distance from the set $\mathrm{x}$ formula is as follows:

$$
\begin{gathered}
\mathrm{y}=[\mathrm{rw} \times \mathrm{p}] \\
\mathrm{X}=-\mathrm{rw} \cdot \mathrm{p}
\end{gathered}
$$

In the above formula, $\mathrm{x}, \mathrm{y}$, and $\mathrm{q}$ represents cutter phase angle function, set $\mathrm{q}$ and combining equation (3), (4), the coordinates of discrete points can be worth a, expressed as (x (i, j), y (i,j), a binary iterative method can not only be q and calculated, but also combine the formula (1), (2) that the corresponding tooth surface radius vector $r_{w}(i, j)$ and $n_{w}(i, j)$ obtained ${ }^{[2]}$.

\section{Determine machine tool error}

Machine adjustment error, tool error, fixture error and force / thermal deformation error in processing is one of several manifestations of the machine errors. Given the thermal deformation error / force generated is difficult to measure it, when the machine rigidity to achieve a certain standard, the error will be smaller, but also correcting machine parameters and tool parameters manner error compensation. Fixture error can be converted into one machine adjustment error. Next, the paper will compare two types of errors.

Tip diameter cutter pressure angle error is the main error performance tool for grinding, the grinding wheel dressing mechanism plays an important role with the installation, if unreasonable wheel dressing method will generate an error when the axis movement; For milling machines, they will have a certain bias when machine tool sharpening adjustment. When using mechanical machining of spiral bevel gears, each parameter corresponds to linear and rotary axes, different positioning accuracy and precision of different axes of movement, whereby the accuracy of the machine determines whether there are differences in error. When using the machine for helical gears CNC machining, CNC axis movement dependent on one or more adjustment parameters, thus, no matter what kind of machine to be clear positioning accuracy and precision of movement, so that machining accuracy is enhanced, reducing tooth error ${ }^{[3]}$.

\section{Calculation method of machine tooth error}

Gear Cutting calculated in accordance with the principle of machine adjustment parameters can be drawn with the tool parameters, combined with the tooth surface mathematical model can determine the tooth surfaces of discrete points, expressed as $(x(i, j), y(i, j))$, discrete points Different points corresponding path vector $r_{w}(i, j)$ and the normal vector $n_{w}(i, j)$. In the same calculation method, you can set the machine to adjust the parameters and error tool parameters, and then the discrete point error derived tooth surface $(x(i, j), y(i, j))$ corresponding to each point of the vector parameters, are 
denoted as $r$ "w (i, j) and n" w (i, j). Gear Measuring Center, CMM for measuring helical bevel gear, the reference point is set to flank the center, represents the theoretical tooth surface and the actual tooth surface there is no error, while the tooth surface at different points in error measurement, along the theoretical tooth surface method sagittal direction close to the actual tooth surface, and then the actual data processing ${ }^{[4]}$, and finally the actual tooth surface normal direction and an error results. Thereby, the midpoint of the corresponding tooth surface is the reference point, denoted as (x $(4,2), y$ $(4,2))$, the first path vector $r_{w}(4,2)$ is calculated, then $r$ "w $(4,2)$ angle to give Each dot diameter vector and normal vector comparison of theoretical tooth surface to be $\mathrm{p}$ and the rotation angle around the axis of the gear, the radius vector $r_{w}(i, j)$ and the normal vector $n_{w}(i, j)$ obtained rotation angle later able to draw new radius vector and normal vector, denoted as $r_{w}{ }_{w}(i, j)$ and normals $n_{w}^{\prime}(i, j)$, then, the theory of tooth surface will coincide with each other and the tooth surface error To so that a more accurate profile error calculation using the optimization algorithm is coordinate rotation algorithm, the variable is set to $q$ with the theoretical tooth surface with normal vector solving, drawn $n_{w}^{\prime}(i, j)$ at the intersection of error and error radius vector $\mathrm{r}{ }_{\mathrm{w}}$, represents the error at this time with the theoretical tooth flank surface normals $n_{w}^{\prime}(i, j)$ direction profile error, namely, e $(i, j)$, specifically expressed as:

$$
e(i, j)=\left(r^{\prime} w(i, j)-r^{\prime \prime} w\right) \cdot n^{\prime} w(i, j)
$$

\section{Discussion on the law of error' $s$ effect on tooth profile}

To gain a more accurate calculation of the profile error, to understand the influence of the error on the tooth, this article describes the different spiral bevel gear cutting method, by using an error analysis software can make the calculation more accurately, its approach is an input parameter variable adjustment - error, by this calculation method provides graphical errors tooth surface and theoretical tooth face position should be displayed, and then come to tooth error. Spiral bevel gears different error influence law of tooth is different, but also there are some similarities. Thus, this paper an example of a spiral bevel gear reducer to reports, the gear machining method using the SGM, the adjustment parameters of the machine, error and tool parameters influence of error on the tooth introduced, secondary parameter gear as follows: tooth surface ratio is expressed bit $i$, the end face modulus $\mathrm{m}_{\mathrm{et}} / \mathrm{mm} 11.6$; axis crossing angle $\sum /\left(^{\circ}\right)$ is 80 ; the average pressure angle size $\alpha_{o} /\left(^{\circ}\right)$ is 10; Ferry midpoint helix angle $\beta \mathrm{m} /\left(^{\circ}\right)$ is 24 ; big wheel tooth surface width $\mathrm{b} / \mathrm{mm}$ is 90 . Processing small round concave and convex machine adjustment parameters and tool parameters:

Processing small round concave radial knife median of $214.56 \mathrm{~mm}$; vertical wheel is $-1.256 / \mathrm{mm}$; round billet mounting angle $13.2456^{\circ}$; horizontal wheel position correction amount is $-8.5624 .2 \mathrm{~mm}$; bed $2.56421 \mathrm{~mm}$; roll ratio of $4.265 \mathrm{~mm}$, basic cradle angle control at $41.2564^{\circ}$.

Ferry surface processing machine: Radial place value $256.217 \mathrm{~mm}$; vertical wheel position $3.25 \mathrm{~mm}$; round billet mounting angle $13.8542^{\circ}$; horizontal wheel position correction amount is $10.524 \mathrm{~mm}$; bed $-2.8541 \mathrm{~mm}$; roll ratio $2.60058 \mathrm{~mm}$, basic cradle angle control is $56.2345^{\circ}$.

To make the comparison method to draw a clearer influence of the tooth, the linear parameters of different error adjustments are made of $0.2 \mathrm{~mm}$ standard error parameters adjusted to different angles of $0.1^{\circ}$, roll ratio error is represented as 0.003 , roll over Amendments II $3 \mathrm{c}$ order coefficient of error of 0.002 , the third-order coefficient ratio correction roller $5 \mathrm{~d}$ error 0.02 , based on the above criteria can be theoretical coefficient tooth surface mapping out the tooth surface figures, fine, there is a difference in error flank dashed theory tooth surface and tooth surface errors are indicated by solid and broken lines, while the combination of thin solid line represents the error is greater than the theoretical tooth flank surfaces, combined with fine dotted line indicates that the error is higher than the theoretical tooth flank surface irregularities in the tooth point position also has a theoretical tooth face opposing the profile error, the unit represented by um $^{[5]}$.

(1).Linear parameter indicates the maximum radial toothed knife drawn influence as an error, but also be able to obtain the level of error and the vertical wheel to wheel. Radial tool position, vertical 
position and round beds, etc. can affect the tooth helix angle, wheel position and determine the level of error is not only the pressure angle there radial tool position, if the radial tool position increases the angle of the spiral decreases; when the bed increases, tooth convex surface helix angle will be smaller; the vertical wheel or gear cutter diameter increases cause an uneven surface helix angle increases; in the bed increases, the tooth convex surface helix angle but minus small, increase the level of wheel position and it will reduce the pressure angle of the uneven surface of the tooth.

(2). Angle design parameters also take into account the factors toothed wheel blank installation angle error is the most important factor, teeth, pressure angle error is also very important in the round billet mounting angle when an error occurs, not only will spiral impact angle, pressure angle tooth more will have an impact, but the impact on the cradle error is small. Round billet mounting angle can be appropriate to increase the tooth convex surface helix angle adjusted to the maximum, then the pressure angle is reduced, but it makes the tooth helix angle convex surface pressure increases; if a single tooth pressure increases also causes tooth convex surface pressure angle becomes larger.

(3). There is a greater impact on the toothed roll over, roll over error is 0.002 , the tooth surface error of $0.1 \mathrm{~mm}$, thus, in the processing machine tools, machine tools requires a combination of practical and theoretical calculation algorithms roll ratio so that the roll The ratio is more accurate.

(4). When second-order coefficient $3 \mathrm{c}$ and third-order coefficient $5 \mathrm{~d}$ error correction, to take into account the factors geodesic torsion, impact on the gear meshing with the direction of the contact area producing area were analyzed; when the machine tool parameter adjustment error or error is negative, will have to change in accordance with the positive or negative changes in tooth analysis, was found to change the trend is in the opposite direction, while the original profile error value substantially constant. In order to verify the calculation results are more accurate analysis results with actual fit of CNC spiral bevel gear grinding machine for an error values are tested during the first grinding process, the use of M \& MSigma6 gear measurement assay. If the test results and analysis of the results of the same, said the processing error and measurement error is small.

\section{Conclusions}

In this paper, spiral bevel gear tooth surface on the basis of a mathematical model, a latest method of calculating an error between the tooth surface and form the theoretical tooth surface error, the machine tool parameters to adjust the parameters and errors are analyzed and then clearly affect tooth law. Visible, do a good job of spiral bevel gear tooth error factors could provide some basis for the fine design tools, and a gear tooth error compensation processing, manufacturing, etc., to promote the safety and efficiency of machine tool manufacturing.

\section{References}

[1] Wang Zhonghou, Li Gang, Aimi Okubo. Spiral bevel gear tooth surfaces contact analysis based on digitized real tooth surfaces. Journal of Mechanical engineering, 2014,50(15):1-11.

[2] Yin Sufeng, Yuan Jingmin. Research of tool adjustment parameter errors on spiral bevel gear tooth surface shape errors. Modern manufacturing engineering, 2011(7):94-97.

[3] Wang Zhiyong, Liu Wei, Zeng Tao. On line measurement based on spiral bevel gears in small tooth-shaped errors of grinding machine. Manufacturing technology and machine tool, 2015(11):122-126.

[4] Li Qiang, Yan Huan, Yan Hongbo. The application of automated visual inspection in logarithmic spiral bevel gears. Modular Machine Tool \& Automatic Manufacturing Technique,2015(4):94-97.

[5] Li Qiang, Wu Shuqin, Yan Hongbo. To establishment of logarithm spiral bevel gear mesh model and fundamental of finite element analysis. Machinery Design and Manufacture, 2011(10):255-257. 